\title{
Effect of Metformin Combined with Losartan on Vascular Endothelial Growth Factor and Angiotensin II in Serum of Spontaneously Hypertensive Rats
}

\author{
J. LUO', MEIZI GUO'1, Y. WU1', C. JIN¹ AND Y. ZHUANG*
}

Department of Cardiology, Shanghai University of Medicine \& Health Sciences, Affiliated Zhoupu Hospital, Shanghai, 201318, ${ }^{1}$ Department of Cardiovascular Surgery, Shanghai General Hospital, Shanghai Jiao Tong University School of Medicine, Shanghai, 200080, P. R. China

Luo et al.: Effect on VEGF and Ang II in serum of SHR

\begin{abstract}
To investigate the effects of metformin combined with losartan on vascular endothelial growth factor and angiotensin II in serum of spontaneously hypertensive rats, 30 spontaneously hypertensive rats were divided into 3 groups according to different treatment methods. The spontaneously hypertensive rats group was administered was without drug intervention. The metformin group was given orally normal saline mixed with metformin tablets. The combination group received the losartan potassium tablets in addition to metformin. The treatment time was $3 \mathrm{w}$ and the expression of vascular endothelial growth factor and angiotensin II in serum was measured. Rats were sacrificed, the brain tissues were taken, the vascular endothelial growth factor and angiotensin II in the brain tissue were detected by immunohistochemistry and immune blotting expression. Before treatment, the blood pressure of rats in the three groups was similar ( $p>0.05)$. Compared to the blood pressure before treatment, the blood pressure of rats in metformin group and combined group decreased significantly $(p<0.05)$. There was no significant change in blood pressure of rats in spontaneously hypertensive rats group. After treatment, the blood pressure level of rats in combined group was lower than that in combined group $(p<0.05)$. The expression of serum vascular endothelial growth factor and angiotensin $I I$ in the 3 groups was similar before treatment $(p>0.05)$. After treatment the levels of vascular endothelial growth factor and angiotensin II in metformin group and combination group decreased $(p<0.05)$. After treatment, the expression of vascular endothelial growth factor and angiotensin II in metformin group was lower than that in spontaneously hypertensive rats group. The expression of angiotensin II in brain tissue of metformin group was less than that of spontaneously hypertensive rats group $(p<0.05)$, and that of the combined group was less than that of metformin group $(p<0.05)$. Combined use in the treatment of spontaneous hypertension has a good antihypertensive effect, and serum and brain tissue vascular endothelial growth factor and angiotensin II were significantly decreased, improving the condition.
\end{abstract}

Key words: SHR, VEGF, Ang II, metformin, losartan

Hypertension is a common clinical disease. At present, 10 to $20 \%$ population of the world are hypertensive, the incidence of which is rising, and it is also the main cause of other heart and brain diseases ${ }^{[1-2]}$. Hypertension is a disease caused by many factors, which can be divided into 2 types, primary and secondary. According to epidemiological survey, more than $90 \%$ of the patients with hypertension are primary. People think that blood vessel is only a simple effector system, but with the deepening of research, it is found that blood vessel itself is a complete organ. While transporting blood, it promotes a variety of local factors to exert vascular tension and other functions ${ }^{[3]}$. Metformin is a common clinical drug of biguanide class. It can reduce blood sugar in diabetes mellitus and has a high utilization rate. However, the literature on the treatment of hypertension with metformin is relatively rare. Losartan is used to treat essential hypertension clinically. It belongs to Angiotensin II antagonist class of drugs and has the function of inhibiting vasoconstriction ${ }^{[4]}$.

Vascular endothelial growth factor (VEGF) is a specific mitotic agent, which can be detected in normal human body and expressed in many organs, such as heart, kidney and brain. As it was known about the relationship of VEGF with various diseases, the relationship between VEGF and hypertension has been studied continuously ${ }^{[5]}$. Angiotensin II (Ang II) is an important component of angiotensin. It exists in both 
adrenal cells and cardiac myocytes. It can bind with angiotensin receptors and produce effects. It can induce vasoconstriction of peripheral blood vessels of patients with hypertension. However, there are few reports about the effects of metformin and losartan on serum levels of VEGF and Ang II in spontaneously hypertensive rats (SHR). In this study, the expression of serum levels of VEGF and Ang II were observed after using the above two drugs in SHR.

Thirty SHR male rats, aged 5-6 w and weighing about 100-150 g, were purchased from Beijing Vitonglihua Company. They were fed sterile diet at room temperature for $2 \mathrm{w}$ in accordance with the Regulations on the Management of Laboratory Animals. Losartan (Beijing Novartis Pharmaceuticals), metformin tablets (Mosadong Company), ELISA Kit (Hualianke Biology), formaldehyde (Taizhou Huayu Company), goat antirabbit first antirabbit (Shanghai Hengyuan Biology). The SHR rats were divided into 3 groups with 10 rats in each group. SHR group was fed normal diet without drug treatment. Metformin group was fed normal diet. Metformin tablets were intragastrically administered with normal saline at a daily dose of $30 \mathrm{~g} / \mathrm{kg}$. The combined group was treated with losartan diluted with normal saline at a daily dose of $30 \mathrm{mg} / \mathrm{kg}$ after $12 \mathrm{w}$ of treatment.

The blood pressure of tail artery in 3 groups of rats was measured before and after treatment. Before and after drug intervention, the orbital venous blood was collected and centrifuged for $2000 \mathrm{rpm}$. The levels of VEGF and Ang II in supernatant were detected by ELISA. Then, anesthetized rats were subjected to craniotomy to extract a small amount of brain tissue after euthanasia. Brain tissues of the 3 groups of rats were fixed with medical formaldehyde, then embedded in paraffin with a thickness of 4 microns, dehydrated with $85 \%$ alcohol, and sealed with goat serum. Goat antirabbit antibody was added, 3,3'-diaminobenzidine was used for the first staining and hematoxylin was used for the second staining. The number of positive cells of VEGF in brain tissue of three groups of rats was observed.

Brain tissue was extracted, lysate was added in a sterile test tube, and mixed it with trypsin extract at 1:100, cooled it in refrigerator for $10 \mathrm{~m}$ to complete cell fission to obtain solution E. Myocardial tissue was taken in Eppendorf tube and $2 \mathrm{ml}$ trypsin extract at 1:100 ratio was added to cause complete cell fission to obtain solution F. Solutions $E$ and $F$ were mixed at 80:1 ratio. The working solution was prepared and kept at $37.5^{\circ}$ in an incubator for $20 \mathrm{~m}$. Glyceraldehyd-3-phosphate dehydrogenase (GAPDH) antibody $(1: 1000)$ was added. Then antiGAPDH and antiGAPDH antibodies were hybridized and chemiluminescent reagents were added. The image is processed by Lab Works software. The results were sorted out by SPSS22.0 software. The correlation between blood pressure changes, the expression levels of VEGF and Ang II in serum and brain tissue before and after treatment was analyzed. The measurement data were calculated by $(X+s)$. T was used between groups and $\mathrm{p}<0.05$ was used to indicate the difference.

Before treatment, the blood pressure levels of the 3 groups were similar ( $>>0.05$ ). Compared with before treatment, the blood pressure of the metformin group and the combined group decreased $(\mathrm{p}<0.05)$. There was no significant change in the blood pressure of the SHR group. After treatment, the blood pressure level of the combined group was lower than that of the metformin group $(\mathrm{p}<0.05)$. As shown in Table 1 .

The serum levels of VEGF and Ang II in the 3 groups were similar before treatment ( $p>0.05)$. Compared with those before treatment, the levels of VEGF and Ang II in the metformin group and the combination group decreased $(p<0.05)$. After treatment, the expression of VEGF and Ang II in metformin group was lower than that in SHR group $(\mathrm{p}<0.05)$, and the expression of these indices in the combined group was lower than that in metformin group $(p<0.05)$. As shown in Table 2 .

The positive expression rate of VEGF was lower in metformin group than in SHR group $(p<0.05)$, and less in combination group than in metformin group $(p<0.05)$. As shown in figs. 1 and 2. The expression of Ang II in brain tissue of metformin group was less than that of SHR group $(p<0.05)$, and that of combined group was less than that of metformin group $(p<0.05)$. As shown in fig 3.

The pathogenesis of spontaneously hypertensive rat model is similar to the pathogenesis of human

TABLE 1: COMPARISON OF BLOOD PRESSURE LEVELS IN THREE GROUPS OF RATS BEFORE AND AFTER TREATMENT

\begin{tabular}{lccc}
\hline Group & $\mathrm{n}$ & $\begin{array}{c}\text { blood pressure } \\
\text { before treatment }\end{array}$ & $\begin{array}{c}\text { blood pressure } \\
\text { after treatment }\end{array}$ \\
\hline SHR group & 10 & $215.79 \pm 5.77$ & $216.32 \pm 6.12$ \\
Metformin group & 10 & $216.78 \pm 5.34$ & $145.57 \pm 5.98^{* \sharp}$ \\
Combined group & 10 & $215.8 \pm 5.69$ & $124.53 \pm 4.94^{* \# \oplus}$ \\
f & & 0.596 & 10.51 \\
P & & 0.556 & $<0.001$ \\
\hline
\end{tabular}

${ }^{*}$ Compared with before treatment, $\mathrm{p}<0.05$; "Compared with SHR group, $\mathrm{p}<0.05$; ${ }^{\circledR}$ Compared with metformin group, $\mathrm{p}<0.05$ 
hypertension. At present, it is an ideal experimental model for preclinical research of this kind of disease. The characteristics of this kind of disease is that the cause of the disease is not clear, but the genetic probability is high. At present, the global incidence of the disease is regional. The United States has the high incidence area of the disease. Studies have confirmed that the patients with essential hypertension are basically asymptomatic, but they need lifelong treatment. In order to realize that they suffer from the disease, some patients cannot effectively control it and involve other organs, at present, clinical treatment mainly relies on past treatment experience and choose a variety of antihypertensive drugs as per the Guidance for Disease Control ${ }^{[6-9]}$.

In this study, it was found that the levels of VEGF in serum and brain tissue of SHR rats increased, while the levels of VEGF and blood pressure decreased in rats treated with metformin. Compared with metformin group, the expression of VEGF and blood pressure in the combined group was lower. Vascular endothelial cell differentiation and potential capillary regeneration can be accelerated when the expression level of vascular endothelial cell is within the normal range in healthy people. However, a large number of studies have shown that there is an important correlation between VEGF and elevated blood pressure. When elevated blood pressure lasts for a short time, the changes of VEGF in patient's serum are small, and there is no pathological change. But when the continuous elevation of blood expression is not effectively controlled, the expression of VEGF is elevated. Now vasoconstriction, resulting in brain tissue lack of blood oxygen supply, leading to a variety of kidney and metabolic diseases ${ }^{[10-12]}$. $\mathrm{Ma}^{[13]}$ study confirmed that patients with essential hypertension exhibited increased insulin resistance, leading to a variety of metabolic diseases, such as hypertension and diabetes mellitus, but metformin treatment of patients with this type of insulin resistance, while reducing blood pressure also reduced the damage to heart and brain tissue, improving hemodynamics ${ }^{[14]}$. Losartan is a drug that can alleviate the progress of blood vessels in patients with hypertension. Zhang and Liang $^{[15]}$ study found that oral absorption of losartan can reduce the level of VEGF in hypertensive patients, reduce the contraction of blood vessels and maintain the stability of organs and tissues. Li et al. ${ }^{[16]}$ study found that metformin combined with losartan can reduce hypertension and the expression of VEGF.

In this study, it was found that the expression of Ang II in serum and tissues of SHR rats increased significantly, blood pressure increased, but was decreased in the metformin group, while Ang II and blood pressure in combination group were even lower. Ang II has a variety of biological reactions, which can increase the volume of extracellular fluid, increase vascular resistance and increase the body's blood pressure. It is mainly divided into two subtypes, AT1 and AT2, which

TABLE 2: COMPARISON OF BLOOD PRESSURE LEVELS IN THREE GROUPS OF RATS BEFORE AND AFTER TREATMENT

\begin{tabular}{lccccc}
\hline \multirow{2}{*}{ Group } & \multirow{2}{*}{$\mathbf{n}$} & \multicolumn{2}{c}{ Ang II } \\
\cline { 3 - 6 } & & Before treatment & After treatment & Before treatment & After treatment \\
\hline SHR group & 10 & $147.49 \pm 25.73$ & $149.49 \pm 23.69$ & $41.26 \pm 4.82$ & $42.76 \pm 4.98$ \\
Metformin group & 10 & $147.51 \pm 25.68$ & $125.89 \pm 12.67^{* \#}$ & $40.35 \pm 4.87$ & $30.67 \pm 3.44^{* \#}$ \\
Combined group & 10 & $148.33 \pm 24.93$ & $117.56 \pm 8.56^{* \# @}$ & $41.33 \pm 4.65$ & $19.85 \pm 2.35^{* \# @}$ \\
$f$ & & 0.089 & 2.11 & 1.739 & 10.06 \\
P Value & & 0.929 & 0.043 & 0.577 & $<0.001$ \\
\hline
\end{tabular}

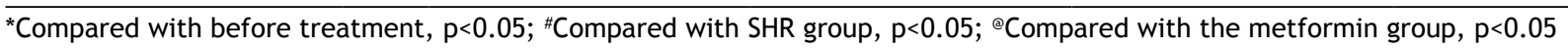
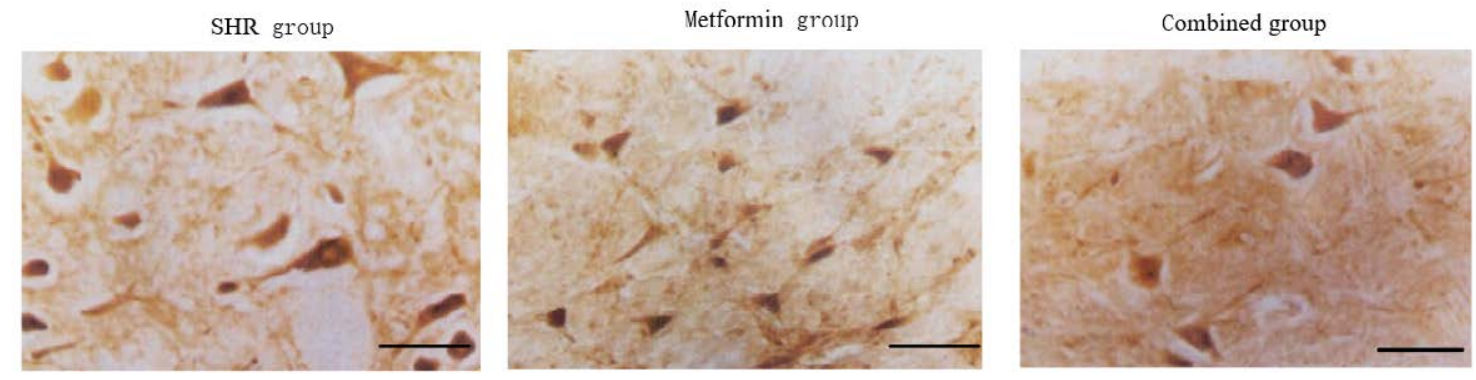

Fig. 1: Expression of vascular endothelial growth factor in brain tissue of the three groups of rats

Positive expression of vascular endothelial growth factor in brain tissue of the SHR group, the metformin group and the combined groups of rats $(200 \times)$ 


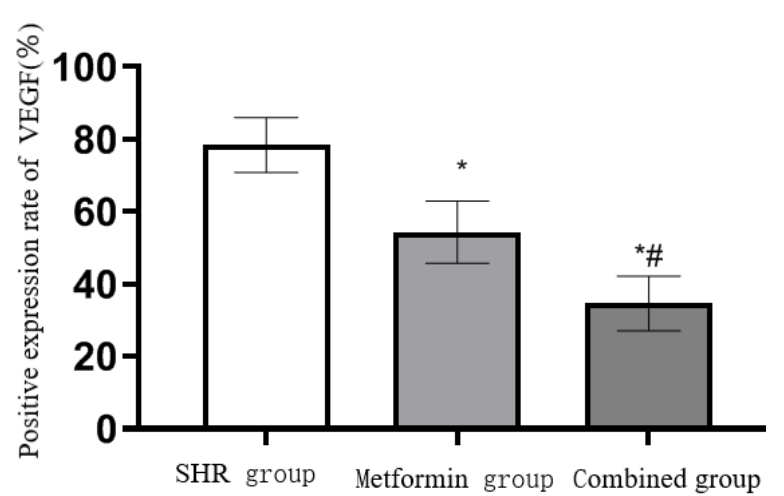

Fig. 2: Expression rate of vascular endothelial growth factor in brain tissue of the three groups of rats

Positive expression rate of vascular endothelial growth factor in brain tissue of the SHR group ( $\square$ ), the metformin group ( $\square$ ) and the combined groups ( $($ ) of rats. *Compared with SHR group, ${ }^{\#}$ Compared with metformin group $\mathrm{p}<0.05$
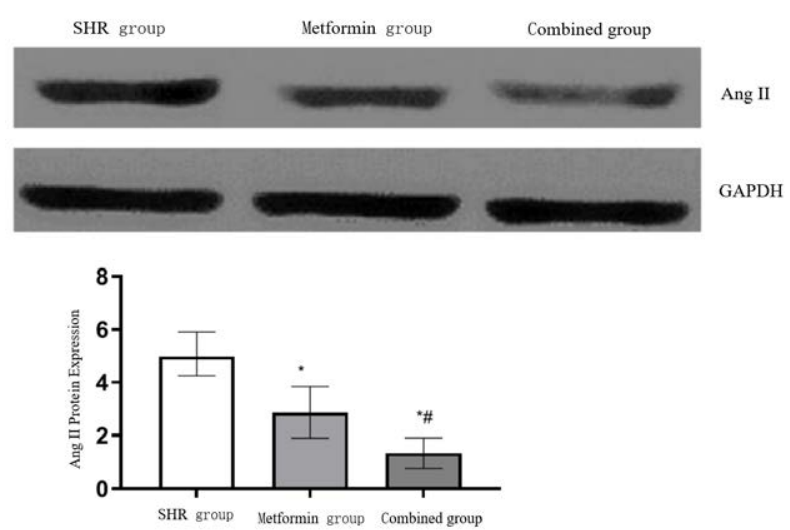

Fig. 3: Ang II expression in the brain tissues of the three groups of rats

Angiotensin II protein expression in the brain tissue of the $(\square)$ SHR group, ( $\square$ ) the metformin group and ( $\square$ ) the combined groups of rats. *Compared with SHR group, ${ }^{*}$ Compared with metformin group $\mathrm{p}<0.05$

play different roles ${ }^{[17,18]}$. Ang II receptors are present in many organs of the body, such as brain and myocardium. It promotes smooth muscle tone by regulating AT1 to exert vasoconstriction and increase the release and absorption of adrenaline. Losartan in SHR antagonizes AT2 receptors blocks the combination of exogenous Ang II and plays a role in lowering blood pressure and alleviated cerebrovascular diseases ${ }^{[19]}$. Wang and Huang ${ }^{[20]}$ study showed that metformin combined with losartan in the treatment of hypertension is more stable, Ang II levels decreased, and widely used in a variety of cardiovascular and cerebrovascular diseases to improve the condition. In summary, metformin in combination with losartan has a good effect in the treatment of spontaneous hypertension, and the levels of serum and brain tissue VEGF and Ang II are significantly decreased, which could improve the condition of patients.

\section{Acknowledgements:}

This work was supported by Key Discipline Groups of Shanghai Pudong New Area (Number PWZxq2017-01).

\section{Conflict of interest:}

No conflict of interest between any of the authors.

\section{REFERENCES}

1. Ding J, Yan HJ, Han XY. Effects of rosuvastatin combined with irbesartan on oxidative stress and serum levels of vascular endothelial growth factor and $\mathrm{CysC}$ in patients with essential hypertension. J Diff Dis 2018;17:18-21.

2. $\mathrm{Hu} \mathrm{LH}, \mathrm{Xie} \mathrm{P}, \mathrm{Chen} \mathrm{DH}$. The effect of combined intervention of medical nutrition and exercise on placental ischemia and hypoxia injury and serum angiogenesis factors in patients with gestational hypertension. J Hainan Med Col 2017;23:2123-26.

3. Wang XY, Chen M, Quan SX. Effect of qinggan jiangya capsule combined with irbesartan on serum adiponectin, vegf and hcy levels in patients with essential hypertension. 2017;17:2068-71.

4. Sun JP, Li JQ, Liao F. Observation on the efficacy of metformin tablets combined with telmisartan in the treatment of obese hypertension patients with impaired glucose tolerance. Shandong Med 2017;57:58-60.

5. $\mathrm{Lu} \mathrm{QJ}, \mathrm{Pu} \mathrm{QH}$, Xiao Y. Effects of glimepiride on blood concentration and antihypertensive effect of losartan and losartan carboxylic acid in patients with type 2 diabetes mellitus and hypertension. Chin Pharm 2018;7:276-8.

6. Jin H, Liu ZJ, Yan CL, Liu FL, Chen L, Zhang QJ, et al. Effects of benazepril and amlodipine on the expression of secretin and somatostatin in spontaneously hypertensive rats. Chin J App Physiol 2018;34:154-8

7. Li DP, Sun DH, Zhang CR. Study on the effect of Tongxinluo Capsule on vascular endothelial function and inflammatory mechanism in spontaneously hypertensive rats. J Clin Pract Hosp 2017;14:241-3.

8. Zhao WB, Li XL, Xi JM. Effects of goushao jiangya granule on Angiotensin II and renal artery calcium sensitive receptor in spontaneously hypertensive rats. Chin Med Emergency 2018;27:793-6.

9. Lu C, Wang KM, Chen WS. Telmisartan and nifedipine on EH patients and their effects on serum levels of HGF and VEGF. Sichuan Med Coll 2018;39:40-3.

10. Li MY, Wang L, Li MX. Role of hypoxia-inducible factor $1 \alpha$ and vascular endothelial growth factor in the pathogenesis of hypoxic pulmonary hypertension in neonatal rats. Chin $\mathrm{J}$ Neonatol 2017;32:64-8.

11. Zhang FH, Wang J, Li J. Effects of Huoxue Tongluo Yiqi decoction combined with metformin on oxidative stress, sinus heart rate turbulence and heart rate variability in patients with essential hypertension complicated with impaired glucose tolerance. J Moder Integr Chin West Med 2018;27:1533-36.

12. Feng TT, Sun P, Wu XX. Effects of hemoperfusion combined with astragalus injection on oxidative stress, neuroendocrine hormones and quality of life in patients with maintenance hemodialysis complicated with refractory hypertension. J Moder Integr Chin West Med 2018;27:48-51.

13. Ma P. Effect of irbesartan and calcium dobesilate on renal hemodynamics in patients with early type 2 diabetes mellitus complicated with hypertension. Hainan Med Coll 2018;29:1055-59.

14. Cheng XP, Gong LH, Li N. Clinical efficacy of EECP combined 
with traditional chinese and western medicine in the treatment of hypertension and its effect on ET, NO and Ang II. Chin Med Emerg 2017;26:481-4.

15. Zhang JJ, Liang WG. Effects of losartan on inflammatory factors, vascular endothelial function and renal function in patients with essential hypertension. Guizhou Med 2017;41:379.

16. Li XD, Yang Y, Zhang L. The effects of losartan potassium on hs-CRP, NO and endothelin-1 in patients with hypertension and diabetes mellitus. Hainan Med Coll 2018;29:13-6.

17. Liu CC, Ma HQ. Clinical efficacy of losartan combined with nifedipine sustained release tablets in the treatment of essential hypertension. J Pract Cardiovasc Pulm Dis 2017;25(7):103-5.

18. $\mathrm{Pu}$ J. Effect of Candesartan and Metformin on left ventricular remodeling and related metabolic parameters in obese hypertensive patients. Chin Cardiovasc Res 2018;16:1127-32.
19. Gong SK. Levamlodipine combined with metformin and rosuvastatin calcium tablets in the treatment of salt-sensitive obesity with mild to moderate hypertension. Prev Treat Cardiovasc Cerebrovasc Dis 2018;18:31-3.

20. Wang JB, Huang YT. Clinical efficacy of losartan and metformin in the treatment of obese hypertension. J Pract Cardiocereb Pulm Vasc Dis 2018;26:132-3.

This is an open access article distributed under the terms of the Creative Commons Attribution-NonCommercial-ShareAlike 3.0 License, which allows others to remix, tweak, and build upon the work non-commercially, as long as the author is credited and the new creations are licensed under the identical terms

This article was originally published in a special issue, "Recent Trends in Biomedical Research"

Indian J Pharm Sci 2020:82(1)spl issue1; XX-XX 